

http://dx.doi.org/10.30681/252610104639

ARTIGO ORIGINAL

\title{
Perfil do câncer do colo uterino e lesões precursoras em um ambulatório de especialidades médicas
}

\section{Profile of uterine cervical and precursing injuries in an ambulatory of medical specialties}

\section{Perfil de cáncer cervical y lesiones precursivas en un ambulatorio de especialidades médicas}

\author{
Giovanna Gomes e Silva1, Lidia Lacerda Furtado², Ana Carla Araújo Campos³, \\ Gabriele Barros de Aviz $^{4}$, Valéria Diniz Calandrini de Azevedo ${ }^{5}$
}

\begin{abstract}
RESUMO
Objetivo: descrever o perfil de pacientes com câncer do colo uterino e lesões precursoras em um ambulatório de especialidades médicas. Método: estudo transversal e quantitativo realizado em Belém, Pará, Brasil. A coleta foi realizada a partir dos prontuários de pacientes diagnosticadas com câncer de colo uterino ou com lesões precursoras no ano de 2018. Foram coletadas informações sociodemográficas e as principais características que são consideradas como fator de risco para desenvolvimento da doença. Resultados: a média de idade das pacientes com câncer foi de 46,3 anos, e 39,3 anos para pacientes com lesões precursoras. A maioria com câncer era solteira $(66,7 \%)$, enquanto aquelas com lesões precursoras eram separadas $(65,7 \%)$. No geral, sem escolaridade (100\% das pacientes com câncer; $66,7 \%$ das com lesões precursoras), multíparas (55,5\%), com coitarca entre 8 e 24 anos. No exame histológico, $69,3 \%$ apresentaram lesões intraepiteliais de alto grau e 30,7\% tiveram câncer. A forma histológica mais frequente foi carcinoma de células escamosas (56,25\%), seguido do adenocarcinoma (43,75\%). Conclusão: idade perimenopausa, baixa escolaridade,
\end{abstract}

\footnotetext{
${ }^{1}$ Médica. Graduada em Medicina pelo Centro Universitário do Estado do Pará. Belém, Pará, Brasil. E-mail: gigomes1@gmail.com ORCID ID: http://orcid.org/0000-0002-4826-2785 Autor para correspondência - Endereço: Avenida Almirante Barroso, no3775 CEP: 66613-903/Souza. Belém, Pará, Brasil.

${ }^{2}$ Médica. Graduada em Medicina pelo Centro Universitário do Estado do Pará. Belém, Pará, Brasil. E-mail: lacerdafurtadolidia@gmail.com ORCID ID: http://orcid.org/0000-0003-4982-1232

${ }^{3}$ Médica. Mestre. Ginecologista e Obstetra. Centro Universitário do Estado do Pará. Belém, Pará, Brasil. E-mail: anacarla.med@outlook.com ORCID ID: http://orcid.org/0000-0001-7375-9729

${ }^{4}$ Médica. Graduada em Medicina pelo Centro Universitário do Estado do Pará. Belém, Pará, Brasil. E-mail: gabrielebaviz@hotmail.com ORCID ID: http://orcid.org/0000-0002-7009-9080

${ }^{5}$ Médica. Graduada em Medicina pelo Centro Universitário do Estado do Pará. Belém, Pará, Brasil. E-mail: valedinizcalandrini@gmail.com ORCID ID: http://orcid.org/0000-0002-5701-197X
}

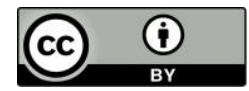

Este artigo está licenciado sob forma de uma licença Creative Commons Atribuição 4.0 Internacional, que permite uso irrestrito, distribuição e reprodução em qualquer meio, desde que a publicação original seja corretamente citada 
coitarca precoce, uso infrequente do preservativo e multiparidade são características das pacientes acometidas pela patologia.

Descritores: Saúde da Mulher; Colo do Útero; Neoplasias do Colo do Útero.

\section{ABSTRACT}

Objective: to describe epidemiological profile of patients with uterine cervical cancer treated at a specialized medical center. Method: cross-sectional and quantitative study conducted in Belém, Pará, Brazil. Data were collected from medical records of patients diagnosed with uterine cervical cancer or precursor lesions in 2018. Sociodemographic information and main characteristics that are considered as risk factors for the development of the disease were collected. Results: the average age of cancer patients was 46.3 years and 39.3 years for patients with precursor lesions. Most cancer patients were single (66.7\%), while those with precursor lesions were divorced (65.7\%). In general, most were illiterate (100\% of cancer patients; $66.7 \%$ of with precursor lesions), multiparous (55.5\%), with first sexual intercourse between 8 and 24 years old. On histological examination, 69.3\% had high-grade intraepithelial lesions and $30.7 \%$ had cancer. The most frequent histological form was squamous cell carcinoma (56.25\%), followed by adenocarcinoma (43.75\%). Conclusion: perimenopause age, low schooling, early coital age, infrequent condom use and multiparity are characteristics of patients affected by the disease.

Descriptors: Women's Health; Cervix Uteri; Uterine Cervical Neoplasms.

\section{RESUMEN}

Objetivo: describir el perfil epidemiológico de pacientes com câncer de cuello uterino y lesiones precursoras tratados en una clínica. Método: estudio transversal y cuantitativo realizado en Belém, Pará, Brasil. La recolección se realizó a partir de los registros médicos de pacientes diagnosticados con cáncer de cuello uterino o lesiones precursoras en 2018, se recopiló información sociodemográfica y las principales características que se consideran un factor de riesgo para el desarrollo de la enfermedad. Resultados: la edad promedio de los pacientes con cáncer fue de 46,3 años y de 39,3 años para los pacientes con lesiones precursoras. La mayoría con cáncer eran solteros (66,7\%), mientras que los que tenían lesiones precursoras estaban separados (65,7\%). En general, sin escolaridad (100\% de pacientes oncológicos; $66,7 \%$ de aquellos con lesiones precursoras), multíparas (55,5\%), con coitarca entre 8 y 24 años. En el examen histológico, el 69,3\% tenía lesiones intraepiteliales de alto grado y el 30,7\% tenía cáncer. La forma histológica más frecuente fue el carcinoma epidermoide (56,25\%), seguido del adenocarcinoma (43,75\%). Conclusión: la edad de la perimenopausia, la baja escolaridad, la edad coital temprana, el uso poco frecuente de condones y la multiparidad son características de las pacientes afectadas por la enfermedad.

Descriptores: Salud de la Mujer; Cuello del Útero; Neoplasias del Cuello Uterino.

\section{INTRODUÇÃO}

O câncer do colo do útero (CCU)

é o segundo tipo de câncer mais frequente entre as mulheres, com aproximadamente 500 mil casos novos por ano no mundo, sendo responsável pelo óbito de aproximadamente $230 \mathrm{mil}$ mulheres/ano1. Em 2018, esperava-se 16.340 casos novos de câncer de colo de útero no Brasil2. 
O CCU é a terceira neoplasia mais incidente entre as mulheres brasileiras $^{3}$. Na Região Norte do Brasil, incluindo o Estado do Pará, o CCU é o mais incidente na população feminina, atrás apenas do câncer de pele não melanoma 4 . Segundo o Instituto Nacional do Câncer (INCA), o Pará apresentou uma taxa de 21,25 casos para cada 100.000 mulheres em $2017^{4}$.

O CCU com exceção do câncer de pele, é o câncer que apresenta maior potencial de prevenção e cura quando diagnosticado precocemente ${ }^{5}$. A prevenção do câncer pode ser realizada pela vacina quadrivalente contra 0 Papiloma Vírus Humano (HPV) 6, 11, 16 e 18, ou através da vacina bivalente contra o HPV 16 e 186. Elas têm mostrado excelente eficácia ao prevenir lesões induzidas por este vírus, já que imuniza contra as formas cancerígenas mais prevalentes ${ }^{7}$.

Atualmente, sabe-se que a infecção por HPV, possui potencial oncogênico, principalmente em relação aos tipos 16 e $18^{6}$, que somado a interação com fatores de risco, como por exemplo, início precoce da vida sexual, múltiplos parceiros sexuais, sexo desprotegido, etilismos e/ou tabagismo, e uso prolongado de anticoncepcionais orais (ACO), justificam o desenvolvimento do câncer de colo de útero $^{8}$. No entanto, a infecção isolada pelo vírus HPV não justifica a oncogênese cervical, mas se considera como um fator predisponente para o desenvolvimento.

Dessa forma, o objetivo deste estudo foi descrever o perfil de pacientes com câncer de colo uterino e lesões precursoras em um ambulatório de especialidades médicas. Essa importância se faz presente, pois dados sobre incidência de doenças direcionam programas estratégicos de prevenção e tratamento, e subsidiam gestores em saúde no processo de investimento, alocação de recursos, definição de metas e avaliação dos resultados ${ }^{8}$.

\section{MÉTODO}

O estudo é do tipo transversal e quantitativo. Foi utilizado o protocolo Strengthening the Reporting of Observational Studies in Epidemiology (STROBE), garantindo o detalhamento adequado do estudo em questão. Todos os aspectos éticos em pesquisas foram respeitados, tendo aprovação do Comitê de Ética em Pesquisa com Seres Humanos (CEP) do Centro Universitário do Estado do Pará (CESUPA), com CAAE: 
08068919.9.0000.5169 e parecer $\mathrm{n}$. 3.172.553.

O estudo foi realizado no Centro de Especialidades Médicas do CESUPA (CEMEC) no ano de 2019, na cidade de Belém, Pará. Foram analisados todos os prontuários nos ambulatórios de Patologia Cervical e de Patologia do Trato Genital Inferior (PTGI) de pacientes atendidas no ano de 2018.

Foram analisados 250 prontuários de pacientes registradas no serviço de Patologia Cervical e no ambulatório de Patologia do Trato Genital Inferior (PTGI) do Centro de Especialidades Médicas. Critérios de inclusão: ter mais de 18 anos, estar regularmente cadastrada no ambulatório e apresentar diagnóstico de câncer de colo de útero ou de lesão precursora confirmado através de biópsia e estudo histopatológico. Utilizou-se prontuários preenchidos completamente ou parcialmente completos desde que incluíssem o exame histopatológico confirmando a patologia. Critérios de exclusão: prontuários com informações rasuradas ou ilegíveis. A amostra final contou com 52 pacientes que contemplavam os critérios do estudo.

Foram coletadas as seguintes variáveis: idade; estado civil; hábitos de etilismo e tabagismo; número de parceiros; idade em que teve a primeira relação sexual; uso de preservativo; gravidez e multiparidade; gravidez precoce; realização de exames preventivos, colposcopia e biopsia e seus respectivos resultados. Ressalta-se que em determinadas variáveis o número da amostra apresenta variação numérica, pois se baseia nos registros dos prontuários.

Os dados obtidos foram analisados e expressos sob a forma de valores mínimo e máximo, média \pm desvio padrão, mediana, percentis e desvio interquartílico e Intervalos de Confiança de $95 \%$. E frequências absoluta e relativa, conforme o caso, sendo apresentado em tabelas.

A normalidade das distribuições foi verificada pelo teste de Normalidade de D’Agostino e Pearson. Para a comparação de duas médias independentes e distribuição simétrica, foi utilizado o teste $t$ de Student para amostras independentes e o teste de Mann-Whitney foi utilizado no caso de distribuições assimétricas. A aderência das distribuições de dados categóricos a uma distribuição teórica foi estimada pelo teste do Qui-Quadrado ou $G$ de aderência. Todos os testes foram executados com o auxílio do programa BioEstat 5.3. Resultados com $p \leq 0.05$ 
(bilateral) foram considerados significativos 9 .

\section{RESULTADOS}

O estudo revelou que no período avaliado a idade média das pacientes com lesões precursoras foi de 39,3 anos e das pacientes com câncer de 46,3 anos. Enquanto $66,7 \%$ das pacientes com câncer era solteira, 65,7\% das pacientes com lesões precursoras era separada. A escolaridade da maioria das pacientes foi não alfabetizada (Tabela 1).

Da amostra, negaram 54,8\% tabagismo e 60,5\% etilismo (Tabela 2). Quanto ao comportamento sexual, a idade média da coitarca foi de 16,1 anos, $48,7 \%$ teve um a três parceiros sexuais e $41,0 \%$ quatro a seis parceiros sexuais. A maioria $(65,4 \%)$ não faz uso regular de preservativo, e 55,6\% teve quatro ou mais gestações (Tabela 3 ).

Quando analisadas sobre a idade na primeira gestação, notou-se que a maioria das pacientes, em média $75 \%$ destas, tiveram sua primeira gestação com média de 21,5 anos $(p=0,0105)$. Sendo que a idade mínima foi de 14 anos e a máxima de 32 anos.

Tabela 1 - Idade, estado civil e escolaridade, segundo a patologia. Belém - PA, Brasil. 2019.

\begin{tabular}{|c|c|c|c|}
\hline Variável & $\begin{array}{l}\text { Câncer } \\
\text { (n; \%) }\end{array}$ & $\begin{array}{l}\text { Lesões precursoras } \\
(\mathrm{n} ; \%)\end{array}$ & p-valor* \\
\hline \multicolumn{4}{|l|}{ Idade (anos) } \\
\hline Média (desvio padrão) & $39,3(10,4)$ & $46,3(9,6)$ & \\
\hline Mín. - Máx. & $23-67$ & $29-63$ & $0,0281^{\dagger}$ \\
\hline \multicolumn{4}{|l|}{ Estado civil } \\
\hline Solteira & $10 ; 66,7^{a}$ & $02 ; 5,7$ & \\
\hline Casada & $05 ; 33,3$ & $10 ; 28,6$ & $<0,0001^{+* *}$ \\
\hline Separada & - & $23 ; 65,7^{a}$ & \\
\hline Total & $15 ; 100$ & $35 ; 100$ & \\
\hline \multicolumn{4}{|l|}{ Escolaridade } \\
\hline Não alfabetizado & $01 ; 100$ & $02 ; 66,7$ & $>0,9999 * *$ \\
\hline Superior incompleto & - & $01 ; 33,3$ & \\
\hline Total & $01 ; 100$ & $03 ; 100$ & \\
\hline
\end{tabular}

*Teste $\mathrm{t}$ de Student para amostras independentes **Teste $\mathrm{G}$ de independência (Análise de Resíduos do Qui-Quadrado) ou Teste Exato de Fisher, conforme o caso. 'Estatisticamente significativo. aFrequência maior que a esperada ao acaso. 
Tabela 2 - Hábitos de vida. Belém - PA, Brasil. 2019.

\begin{tabular}{lccc}
\hline Variável & $\mathbf{n}$ & $\%$ & p-valor* \\
\hline Tabagismo $(\mathbf{n = 4 2})$ & 05 & 11,9 & \\
$\quad$ Fumante & 14 & 33,3 & $0,0032^{\dagger}$ \\
$\quad$ Ex-fumante & 23 & $54,8^{\mathrm{a}}$ & \\
$\quad$ Nega & & & \\
Etilismo $(\mathbf{n = 3 8 )}$ & 12 & 31,6 & $0,0004^{\dagger}$ \\
$\quad$ Etilista & 03 & 7,9 & \\
$\quad$ Ex-etilista & 23 & $60,5^{\mathrm{a}}$ & \\
$\quad$ Nega & & & \\
Frequência ingesta de álcool (n=11) & 03 & 27,2 & 0,2021 \\
1 a 2 vezes/semana & 04 & 36,4 & \\
1 a 2 vezes/mês & 04 & 36,4 & \\
3 a 4 vezes/mês & 04,4
\end{tabular}

${ }^{*}$ Qui-Quadrado de aderência. ${ }^{\dagger}$ Estatisticamente significativo. a Frequência maior que a esperada ao acaso.

Tabela 3 - Comportamento sexual e perfil obstétrico. Belém - PA, Brasil. 2019.

\begin{tabular}{|c|c|c|c|}
\hline Variável & $\mathrm{n}$ & $\%$ & p-valor* \\
\hline \multicolumn{4}{|c|}{ Coitarca (anos) } \\
\hline Idade Média & \multirow{2}{*}{\multicolumn{2}{|c|}{$\begin{array}{c}16,1 \\
8-24\end{array}$}} & \\
\hline Mín. - máx. & & & 0,0816 \\
\hline \multicolumn{4}{|c|}{ Parceiros sexuais na vida $(n=39)$} \\
\hline $1|-| 3$ & 19 & $48,7^{\mathrm{a}}$ & $<0,0001^{\dagger}$ \\
\hline $4|-| 6$ & 16 & $41,0^{\mathrm{a}}$ & \\
\hline $7|-| 9$ & 01 & 2,6 & \\
\hline$\geq 10$ & 03 & 7,7 & \\
\hline \multicolumn{4}{|c|}{ Parceiros sexuais no último ano $(n=30)$} \\
\hline Nenhum & 06 & 20,1 & $<0,0001^{\dagger}$ \\
\hline $1|-| 2$ & 22 & $73,3^{\mathrm{a}}$ & \\
\hline $3|-| 4$ & 01 & 3,3 & \\
\hline$\geq 5$ & 01 & 3,3 & \\
\hline \multicolumn{4}{|c|}{ Parceiros sexuais novos no último ano $(n=36)$} \\
\hline Nenhum & 21 & $58,4^{\mathrm{a}}$ & $<0,0001^{\dagger}$ \\
\hline $1|-| 2$ & 12 & 33,3 & \\
\hline $3|-| 4$ & 01 & 2,7 & \\
\hline$\geq 5$ & 02 & 5,6 & \\
\hline \multicolumn{4}{|c|}{ Uso de preservativo $(n=26)$} \\
\hline Sempre & 03 & 11,5 & \\
\hline Às vezes & 17 & $65,4^{a}$ & $0,0020^{\dagger}$ \\
\hline Nunca & 06 & 23,1 & \\
\hline \multicolumn{4}{|c|}{ Gestações (n=45) } \\
\hline Nenhuma & 02 & 4,4 & $<0,0001^{\dagger}$ \\
\hline 1 a 3 & 18 & $40,0^{\mathrm{a}}$ & \\
\hline 4 ou mais & 25 & $55,6^{\mathrm{a}}$ & \\
\hline \multicolumn{4}{|l|}{ Partos $(n=43)$} \\
\hline $1|-| 3$ & 21 & $48,8^{\mathrm{a}}$ & \\
\hline $4|-| 6$ & 14 & 32,6 & \\
\hline $7|-| 9$ & 06 & 13,9 & $0,0002^{\prime}$ \\
\hline 10 & 02 & 4,6 & \\
\hline
\end{tabular}




\begin{tabular}{cccc}
\hline Abortamento $(\mathrm{n}=43)$ & 28 & $65,1^{\mathrm{a}}$ & \\
0 & 13 & 30,2 & $<0,0001^{\dagger}$ \\
1 & 02 & 4,7 & \\
2 & 02 & \\
\hline
\end{tabular}

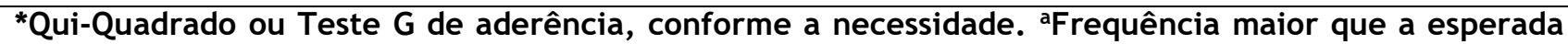
ao acaso. ${ }^{\dagger}$ Estatisticamente significativo.

A seguir são apresentados dados quanto ao histórico das pacientes, sendo $63 \%$ sem uso de contraceptivo hormonal oral. A maioria refere quadro prévio de corrimento, prurido ou irritação vaginal (54,1\%), porém $84,6 \%$ nega histórico de infecção sexualmente transmissíveis (IST) (Tabela 4).

Dentre os resultados de exames, $69,4 \%$ da colpocitologia oncótica teve resultado suspeito, enquanto $88,3 \% \mathrm{da}$ colposcopia teve resultado anormal, nos exames histopatológicos, a maioria $(69,3 \%)$ correspondeu a lesões precursoras do câncer, estas lesões todas tiveram como descrição lesão intraepitelial de alto grau (HSIL) ou carcinoma in situ. As demais pacientes, 30,7\% tiveram o câncer como diagnóstico, sendo 56,25\% com carcinoma de células escamosas e 43,75\% com adenocarcinoma (Tabela 5).

Tabela 4 - Uso de anticoncepcional, histórico de infecção sexualmente transmissível e corrimento entre as pacientes atendidas no Serviço de Patologia Cervical ou Patologia do Trato Genital Inferior. Belém - PA, Brasil. 2019.

\begin{tabular}{|c|c|c|c|}
\hline Variável & $\mathbf{N}$ & $\%$ & p-valor* \\
\hline \multicolumn{4}{|c|}{ Uso de contraceptivo hormonal oral $(n=27)$} \\
\hline Já usou & 07 & 25,9 & \multirow{3}{*}{$0,0032^{\dagger}$} \\
\hline Usa atualmente & 03 & 11,1 & \\
\hline Nega & 17 & $63,0^{a}$ & \\
\hline \multicolumn{4}{|c|}{ História de corrimento/prurido/irritação vaginal $(n=37)$} \\
\hline Sim & 20 & 54,1 & \multirow{2}{*}{0,7428} \\
\hline Não & 17 & 45,9 & \\
\hline \multicolumn{4}{|c|}{ História de IST $(n=39)$} \\
\hline Sim & 06 & 15,4 & \multirow{2}{*}{$<0,0001^{\dagger}$} \\
\hline Não & 33 & $84,6^{a}$ & \\
\hline
\end{tabular}


Tabela 5 - Resultados dos exames realizados pelas pacientes. Belém - PA, Brasil. 2019.

\begin{tabular}{|c|c|c|c|}
\hline Variável & $\mathrm{n}$ & $\%$ & p-valor* \\
\hline \multicolumn{4}{|l|}{ Colpocitologia oncótica $(n=49)$} \\
\hline Normal & 07 & 14,4 & \multirow{5}{*}{$<0,0001^{\dagger}$} \\
\hline Alterado Suspeito (ASC-H, AGUS, HSIL, Carcinoma in situ) & 34 & $69,4^{\mathrm{a}}$ & \\
\hline Alterado (LSIL e ASC-US) & 04 & 8,1 & \\
\hline Carcinoma invasor & 04 & 8,1 & \\
\hline Total & 49 & 100 & \\
\hline \multicolumn{4}{|l|}{ Resultado detalhado da Colpocitologia oncótica $(n=49)$} \\
\hline AGUS & 02 & 4,1 & \multirow{9}{*}{$<0,0001^{\dagger}$} \\
\hline ASC-H & 04 & 8,2 & \\
\hline ASC-US & 03 & 6,1 & \\
\hline ASC-US + AGUS & 01 & 2,0 & \\
\hline LSIL & 01 & 2,0 & \\
\hline HSIL & 27 & $55,1^{a}$ & \\
\hline Carcinoma escamoso/Adenocarcinoma invasor & 04 & 8,2 & \\
\hline Sem lesão/Alterações Inflamatórias & 07 & 14,3 & \\
\hline Total & 49 & 100 & \\
\hline \multicolumn{4}{|l|}{ Colposcopia geral $(n=43)$} \\
\hline Achados normais & 02 & 4,7 & \multirow{4}{*}{$<0,0001^{\dagger}$} \\
\hline Achados anormais & 38 & 88,3 & \\
\hline Achados com suspeita de invasão & 03 & 7,0 & \\
\hline Total & 43 & 100 & \\
\hline \multicolumn{4}{|l|}{ Resultado detalhado colposcopia $(n=41)$} \\
\hline Alteração grau I & 11 & 26,8 & \multirow{5}{*}{$<0,0001^{\dagger}$} \\
\hline Alteração grau II & 26 & 63,4 & \\
\hline Alterações grau I e II & 01 & 2,5 & \\
\hline Suspeita de invasão & 03 & 7,3 & \\
\hline Total & 41 & 100 & \\
\hline \multicolumn{4}{|l|}{ Resultado do exame histológico } \\
\hline Carcinoma escamoso/adenocarcinoma & 16 & 30,7 & \multirow{3}{*}{0,0084} \\
\hline HSIL & 36 & 69,3 & \\
\hline Total & 52 & 100 & \\
\hline \multicolumn{4}{|l|}{ Resultado do tipo histológico do câncer } \\
\hline Carcinoma de células escamosas & 09 & 56,25 & \multirow{3}{*}{0,8026} \\
\hline Adenocarcinoma & 07 & 43,75 & \\
\hline Total & 16 & 100 & \\
\hline
\end{tabular}

*Qui-Quadrado ou Teste G de aderência, conforme a necessidade. Células glandulares atípicas de significado indeterminado (AGUS). Células escamosas atípicas de significado indeterminado possivelmente não neoplásicas (ASC-US). Células escamosas atípicas não sendo possível excluir lesão intraepitelial de alto grau (ASC-H). Lesão intraepitelial (LIE). 'Estatisticamente significativo. ${ }^{a}$ Frequência maior que a esperada ao acaso.

\section{DISCUSSÃO}

A média da idade das pacientes com câncer foi de 46,3 anos, compatível com outros estudos ${ }^{10,11}$, que tiveram a faixa de 40-59 anos a mais acometida pelo CCU. Enquanto a idade média das pacientes com lesões precursoras avaliadas pelo presente estudo foi de 39,3 anos, semelhante a demonstrada 
por um estudo recente ${ }^{12}$. Na Bahia ${ }^{13}$ as pacientes com lesões precursoras tinham entre 20 a 39 anos. As pacientes com CCU tiveram a média da idade sete anos maior do que aquelas com lesões precursoras.

Um estudo ${ }^{14}$ realizado em Pernambuco mostra que a maioria das mulheres com CCU são solteiras, assim como observado neste estudo, mas difere da realidade nacional ${ }^{15} \mathrm{em}$ que a maioria era casada. Em pacientes com lesões precursoras prevaleceu a condição de divorciadas. A escolaridade das participantes foi baixa, semelhante a outros estudos ${ }^{14,15}$, que mostram que as mulheres acometidas pela doença têm problemas no seguimento dos estudos, e boa parte não são alfabetizadas.

No presente estudo, apenas $11,9 \%$ das pacientes eram tabagistas. 0 tabagismo aumenta o risco de progressão do tumor. 0 risco é maior quanto mais precoce for a introdução do fumo, e é proporcional ao número de cigarros fumados por dia. Um estudo mineiro encontrou metabólitos do cigarro no muco cervical de pacientes tabagistas com CCU em níveis mais elevados do que no sangue, indício da participação do tabaco na gênese da doença ${ }^{16}$.

A maioria das pacientes negou 0 etilismo $(60,5 \%)$. O etilismo não está diretamente associado ao risco de câncer de colo de útero, porém o hábito de ingerir bebidas alcóolicas está muito relacionado ao tabagismo, por isso são investigados de forma conjunta ${ }^{16,17}$.

No presente estudo, a idade média da coitarca foi aos 16 anos. Em Portugal $^{18}$ a maioria de mulheres que desenvolveram a doença, iniciaram a atividade sexual antes dos 18 anos. Sabe-se que quanto mais precoce o início da vida sexual, mais prolongada ela se torna, e favorece o aumento do número de parceiros, logo, a mulher se torna mais exposta a situações de risco, como no contato com o vírus do $\mathrm{HPV}^{6}$. Sendo assim, o início precoce da atividade sexual está relacionado a infecção por HPV, já que o epitélio imaturo se encontra mais susceptível a lesões oncogênicas ${ }^{18}$.

Apenas $25 \%$ das pacientes neste estudo fazem uso de anticoncepcionais orais (ACO). Os ACO são associados a oncogênese, já que interferem no metabolismo celular e induzem a exposição do epitélio glandular aos agentes agressores ${ }^{19}$. Estudo realizado em Santa Catarina ${ }^{19}$ afirma que mulheres em uso do ACO têm diminuição das IgA e lgG, o que propicia um meio favorável ao aparecimento de lesões por HPV. 
Neste trabalho, 55,6\% tiveram quatro ou mais gestações. Em outro estudo $^{14} 73,44 \%$ das mulheres com diagnóstico de CCU tiveram três ou mais gestações. Investigação semelhante mostra que a ausência de filhos diminui a sobrecarga da mulher e permite que ela utilize seu tempo para cuidar de si, estudar e realizar exames preventivos, como o papanicolau ${ }^{20}$.

A maioria das participantes não possui história prévia de alguma IST. Embora, é consenso que a infecção pelo HPV geralmente é assintomática ${ }^{19,21}$, ou com lesões microscópicas que passam despercebidas. Quando a infecção por HPV cursa com condiloma acuminado, está relacionada frequentemente a subtipos não oncogênicos, como o HPV 6 e $11^{19,21,22}$.

0 rastreio para detecção das lesões precursoras de CCU e o diagnóstico do câncer em sua fase inicial é possível por meio da realização periódica do exame citopatológico ${ }^{3}$. Apenas $14,4 \%$ das pacientes tinham o exame de colpocitologia oncótica sem alteração. Isto demonstra a importância da realização destes exames, já que após investigações todas foram diagnosticadas com CCU ou lesões precursoras.
Em estudo semelhante ${ }^{14}, \quad 0$ resultado de HSIL foi o mais frequente nos exames colpocitologia oncótica avaliados $(45,6 \%)$, concordando com o presente estudo, em que a frequência de HSIL foi de 55,1\% no exame de colpocitologia oncótica. Na colposcopia o resultado mais frequente foi de achados anormais $(88,3 \%)$ com alterações grau II $(63,4 \%)$.

No exame histológico, $69,3 \%$ apresentaram lesões intraepiteliais de alto grau e 30,7\% tiveram CCU. A forma histológica mais frequente entre as pacientes foi carcinoma de células escamosas $(56,25 \%)$, resultado similar ao encontrado no Rio de Janeiro (RJ) ${ }^{11}$ que também teve a predominância desta forma, com frequência de $80 \%$. Em seguida está o adenocarcinoma (43,75\%), este tipo histológico também estava em segundo lugar dos mais frequentes no mesmo estudo ${ }^{11}$, porém com uma representatividade menor, correspondendo a $15 \%$ dos casos.

Vale ressaltar que este estudo apresenta as limitações inerentes aos vieses inerentes ao estudo transversal, apesar dos esforços em evitá-los; as buscas com base em prontuários, que nem sempre contêm dados completos. Ainda, pode mencionar a ausência de 
exploração integral dos fatores de risco associados ao CCU.

\section{CONCLUSÃO}

As

características

epidemiológicas mais frequentes relacionadas ao CCU e as lesões precursoras são semelhantes, como idade perimenopausa, baixa escolaridade, coitarca precoce, uso infrequente do preservativo e multiparidade. A média de idade das pacientes com CCU foi maior que a média as pacientes com lesões precursoras, este dado mostra 0 desenvolvimento natural da doença, em que as lesões precursoras surgem em pacientes mais jovens, e se não forem tratadas podem evoluir para o câncer no decorrer da vida.

O caráter progressivo do $\mathrm{CCU}$, que inicia com lesões precursoras facilmente detectáveis pelo exame de rastreio, torna a realização periódica do exame citopatológico imprescindível para a detecção precoce da patologia, além de possibilitar tratamento, cura e aumento de sobrevida. Diante disso, os achados do estudo têm relevância na implementação de ações públicas para pacientes com perfis semelhantes, para que seja realizado rastreio e diagnóstico precoce.

\section{REFERÊNCIAS}

1. Ministério da Saúde (BR). Instituto Nacional de Câncer. Estimativa de Câncer no Brasil, 2016. Rio de Janeiro: INCA; 2018.

2. Organização Pan-Americana De Saúde. Controle integral do câncer do colo do útero: Guia de práticas essenciais. Washington: OPAS; 2018.

3. Mesquita AD, Teles KKN, Silva SCB, Silva FR, Lima LKC, Costa RSL, et al. Conhecimentos, atitudes e práticas de mulheres frente ao exame preventivo do câncer do colo uterino. J Health NPEPS. 2020; 5(1):261-275.

4. Ministério da Saúde (BR). Instituto Nacional de Câncer José Alencar Gomes da Silva. Estimativa 2018: incidência de câncer no Brasil. Coordenação de Prevenção e Vigilância. - Rio de Janeiro: INCA; 2017.

5. Favaro CRP, Durant LC, Paterra TSV, Panobianco MS, Gozzo TO. Perfil epidemiológico de mulheres com câncer de colo do útero tratadas em hospital terciário. Rev Enferm CentOeste Min. 2019; 9:e3253. 
6. Costa TML, Heráclio S, Amorim MMR, Souza PRE, Lubambo N, Souza GFA, et al. Papilomavírus humano e fatores de risco para adenocarcinoma cervical no estado de Pernambuco, Brasil. Rev Bras Saúde Mater Infant. 2019; 19(3):641-649.

7. Carvalho AMC, Andrade EMLR, Nogueira LT, Araújo TME. Adesão à vacina hpv entre os adolescentes: revisão integrativa. Texto contexto enferm. 2019; 28:e20180257.

8. Vaz GP, Bitencourt EL, Martins GS, De Carvalho $A A B$, Reis Júnior PM. Perfil epidemiológico do câncer de colo de útero na região norte do brasil no período de 2010 a 2018. Rev Patol Tocantins. 2020; 7(2).

9. Ayres M, Ayres Jr M, Ayres DL, Santos AS. BioEstat 5.4. Aplicações estatísticas nas áreas das ciências biológicas e médicas. Belém: Mamirauá; 2015.

10. Moreira RCR, Souza LO, Carvalho MAS, Saldanha SCS. Perfil Epidemiológico Do Câncer De Colo Uterino No Município De Feira De Santana, Bahia, Brasil. J Nursing UFPE on line. 2019; 3(4):998-1004.

11. Rozario S, Silva IF, Koifman RJ, Silva IF. Caracterização de mulheres com câncer cervical atendidas no Inca por tipo histológico. Rev Saúde Pública. 2019; 53:88.

12. Pedrosa TFM, Magalhães Filho SD, Peres AL. Perfil das mulheres com alterações cervicais em uma cidade do nordeste brasileiro. J Bras Patol Med Lab. 2019; 55(1):32-43.

13. Mattos PSMS. Frequência de neoplasia intraepitelial cervical nas pacientes que realizaram exame de papanicolau atendidas no sistema único de saúde na Bahia. Rev Eletrônica Atualiza Saúde. 2016; 3(3):07-14.

14. Silva RCG, Silva ACO, Peres AL, Oliveira SR. Perfil de mulheres com câncer de colo do útero atendidas para tratamento em centro de oncologia. Rev Bras Saúde Mater Infant. 2018; 18(4):695-702.

15. Renna Júnior NL, Silva GA. Tendências temporais e fatores associados ao diagnóstico em estágio avançado de câncer do colo uterino: análise dos dados dos registros hospitalares de câncer no Brasil, 2000-2012. Epidemiol Serv Saúde. 2018; 27(2):e2017285.

16. Treco IC. Prevalência de alterações citopatológicas no colo uterino em mulheres de um município do sudoeste paranaense e potenciais fatores de risco. Francisco Beltrão. 
Dissertação [Mestrado] - Programa de Pós-graduação Stricto Sensu em Ciências Aplicadas à Saúde, Universidade Estadual do Oeste do Paraná; 2019.

17. Barezzi MB, Pereira CV, Cruz DT, Leite ICS. Prevalência e fatores associados ao consumo de álcool e de tabaco em idosos não institucionalizados. Rev bras geriatr gerontol. 2018; 21(2):123-133.

18. Ramalho SMF. A educação para a saúde na prevenção do cancro do colo do útero nas mulheres em idade adulta. Lisboa. Dissertação [Mestrado] - Programa de Mestrado da Escola Superior de Enfermagem de Lisboa; 2018.

19. Nunes R, Siqueira I, Traebert J. Associação entre contracepção oral com etinilestradiol $e$ as lesões induzidas pelo HPV no colo uterino. Arq Catarin Med. 2017; 46(4):128-139.
20. Melo EMF, Linhares FMP, Silva TM, Pontes CM, Santos AHS, Oliveira SC. Câncer cervico-uterino: conhecimento, atitude e prática sobre o exame de prevenção. Rev Bras Enferm. 2019; 72(Suppl 3):2531.

21. Abreu MNS, Soares AD, Ramos DAO, Soares FV, Nunes Filho G, Valadão $A F$, et al. Conhecimento e percepção sobre o HPV na população com mais de 18 anos da cidade de Ipatinga, MG, Brasil. Ciênc Saúde Colet. 2018; 23(3):849-860.

22. Rocha SMM, Bahia MO, Rocha CAM. Perfil dos exames citopatológicos do colo do útero realizados na Casa da Mulher, Estado do Pará, Brasil. Rev Pan-Amaz Saude. 2016; 7(3):51-55.

Financiamento: Os autores declaram que não houve financiamento.

Conflito de interesses: Os autores declaram não haver conflito de interesses.

Participação dos autores:

- Concepção: Silva GG, Furtado LL, Campos ACA, Aviz GB, Azevedo VDC.

- Desenvolvimento: Silva GG, Furtado LL, Campos ACA, Aviz GB, Azevedo VDC.

- Redação e revisão: Silva GG, Furtado LL, Campos ACA, Aviz GB, Azevedo VDC.

Como citar este artigo: Silva GG, Furtado LL, Campos ACA, Aviz GB, Azevedo VDC. Perfil do câncer do colo uterino e lesões precursoras em um ambulatório de especialidades médicas. J Health NPEPS. 2020; 5(2):119-131. 